\title{
DETERMINAÇÃO DO COMPRIMENTO DA ESTACA PARA A PRODUÇÃO DE MUDAS DE PITAIA (Hylocereus costaricensis) EM AMBIENTE PROTEGIDO
}

\author{
Antonio Robson Moreira ${ }^{1 *}$, Fernanda Ludmyla Barbosa de Souza ${ }^{2}$, Raimundo Thiago Lima da Silva ${ }^{3}$, Raimundo Leonardo Lima de \\ Oliveira $^{4}$, Airton dos Santos Alonço ${ }^{1}$, Cândido Ferreira de Oliveira Neto ${ }^{3}$, Samara Ketely Almeida de Sousa ${ }^{3}$ \\ ${ }^{1}$ Departamento de Engenharia Rural, Universidade Federal de Santa Maria, 97105-900, Santa Maria, Brasil. \\ ${ }^{2}$ Departamento de Agronomia, Universidade Estadual do Oeste do Paraná, 85960-000, Marechal Cândido Rondon, Brasil. \\ ${ }^{3}$ Departamento de Agronomia, Universidade Federal Rural da Amazônia, 68650-000, Capitão Poço, Brasil. \\ ${ }^{4}$ Departamento de Solos e Adubos, Universidade Estadual Paulista, 14884-900, Jaboticabal, Brasil. \\ *E-mail: robson.n.13@hotmail.com
}

\section{RESUMO}

O objetivo deste trabalho foi determinar o melhor comprimento da estaca para a produção de mudas de pitaia (Hylocereus costaricensis) em ambiente protegido. O experimento foi realizado na Universidade Federal Rural da Amazônia, Campus Capitão Poço - Pará, em delineamento inteiramente casualizado com cinco tratamentos e quatro repetições, sendo cada tratamento formado por um comprimento de estacas: $10 \mathrm{~cm}, 15 \mathrm{~cm}, 20 \mathrm{~cm}, 25 \mathrm{~cm}$ e $30 \mathrm{~cm}$, formando 20 parcelas experimentais. Cada uma dessas parcelas foi composta por quatro estacas, totalizando 80 mudas. Com o surgimento dos primeiros brotos, deu-se início à avaliação dos seguintes parâmetros: altura da muda em $\mathrm{cm}(\mathrm{AL})$, número de brotos $(\mathrm{NB})$, comprimento e diâmetro do primeiro broto emitido (CPB e DBI) e comprimento do segundo broto (CSB). Os resultados obtidos comprovam a eficácia da utilização de menores comprimentos de estacas, principalmente, quando ocorre escassez de material propagativo, já que o ponto de máxima emissão de brotações se deu próximo a estacas de $25 \mathrm{~cm}$. No entanto, se houver abundância, estacas maiores podem proporcionar incrementos superiores em altura, diâmetro e comprimento das brotações emitidas. O comprimento das estacas influenciou ganho em altura, comprimento e diâmetro das brotações emitidas, apresentando uma relação positiva linear, ou seja, quanto maior a estaca, maiores os incrementos. Estacas de 25 $\mathrm{cm}$ podem ser utilizadas para a produção de mudas sem comprometer o número de brotações emitidas. O ponto de máxima emissão de brotações se deu em $24,11 \mathrm{~cm}$, quando as mudas poderiam emitir, aproximadamente, três brotações.

Palavras-chave: Propagação vegetativa, Cladódio, Cactaceae, Metabolismo CAM.

\section{Introdução}

O Brasil é um dos maiores produtores mundiais de frutas e, ainda, tem potencial para expandir sua produção, uma vez que existem inúmeras espécies nativas e exóticas, não exploradas comercialmente ou pouco exploradas. Dentre elas, encontra-se a pitaia, considerada, no país, uma fruta exótica, apesar de existirem espécies nativas no cerrado e matas de transição, conhecidas, popularmente, como pitaia-do-cerrado ou "saborosa"[1].

Originária das florestas tropicais do México, América Central e América do Sul [2-3], pertencente à família Cactaceae, a pitaia apresenta características como: perene de hábito epífito, raízes adventícias, cladódio triangular, flor hermafrodita, antese noturna, polpa firme e sabor adocicado [45]. Possui, também, propriedades medicinais, auxiliando no processo digestivo, tem efeito benéfico contra gastrites, prevenção contra o câncer do cólon e diabetes, neutraliza substâncias tóxicas, como os metais pesados, reduz os níveis de colesterol e as altas pressões do sangue, além de os cladódios e as flores serem usados contra problemas renais. Pode produzir logo no primeiro ano após o plantio [6-7] e atingir produtividade de $34 \mathrm{t} \mathrm{ha}^{-1}$, como ocorrido em Israel no quinto ano de cultivo [8].

Apesar de todas essas vantagens, não existe, no mercado, uma cultivar que atenda os anseios de clima e produtividade. As mudas comercializadas hoje são provenientes de matrizes não selecionadas, o que provoca a formação de pomares heterogêneos [9]. Portanto, para formar pomares mais homogêneos, a propagação por estaquia é o método mais recomendado [10]. Esses mesmos autores encontraram resultados satisfatórios na formação de mudas sob telado, utilizando estacas de 15 e $25 \mathrm{~cm}$ [10]. Outros autores afirmam que estacas de 50 e $70 \mathrm{~cm}$ podem ser enraizadas diretamente no campo [7]. Tais resultados são bastante amplos no que diz respeito ao comprimento da estaca, fator que pode influenciar 
tanto nas reservas de carboidratos como no volume de auxinas endógenas, o que proporciona maior sobrevivência, emissão mais rápida de raízes e brotos durante o crescimento inicial [11$13]$.

Diante do exposto em relação à ampla faixa de recomendação quanto ao comprimento da estaca para a formação de mudas, este trabalho teve como objetivo determinar o comprimento da estaca para a produção de mudas de pitaia em ambiente protegido.

\section{Parte Experimental}

\subsection{Localização da área do estudo}

$\mathrm{O}$ experimento foi realizado na área experimental da Universidade Federal Rural da Amazônia, campus Capitão Poço Pará (UFRA-CCP), com coordenadas geográficas de latitude $01^{\circ} 44^{\prime} 47^{\prime}$ ' $\mathrm{S}$ e longitude $47^{\circ} 03$ '34' W, em estufa plástica com dimensões $16 \mathrm{~m} \times 30 \mathrm{~m}$. Foi executado no período de 15 de abril a 30 de agosto de 2014.

\subsection{Material e métodos}

O material propagativo foi proveniente de plantas matrizes de cinco anos de idade aproximadamente. $\mathrm{O}$ delineamento experimental utilizado foi $\mathrm{o}$ inteiramente casualizado (DIC), com cinco tratamentos e quatro repetições, sendo cada tratamento formado por um comprimento de estacas: $10 \mathrm{~cm}, 15 \mathrm{~cm}, 20 \mathrm{~cm}, 25 \mathrm{~cm}$ e $30 \mathrm{~cm}$, formando 20 parcelas experimentais, cada uma dessas constituída por quatro estacas, totalizando 80 mudas. Cada estaca foi comportada individualmente em saco plástico próprio para mudas, com capacidade para 1 litro de substrato feito à base de esterco bovino, terra preta e casca de arroz carbonizada, na proporção de $1: 1: 1$.

Com o auxílio de uma fita métrica e um paquímetro digital de precisão $0,01 \mathrm{~mm}, 27$ dias após a implantação do experimento, com o surgimento dos primeiros brotos, deu-se início à avaliação dos seguintes parâmetros da parte aérea: altura da muda em cm (AL), número de brotos (NB), comprimento e diâmetro do primeiro broto emitido pela muda (CPB e DBI) e comprimento do segundo broto (CSB). As medições foram realizadas de 10 em 10 dias. A irrigação foi realizada manualmente com turno de rega de $48 \mathrm{~h}$, sempre deixado o solo na capacidade de campo. A primeira adubação foi realizada três meses após o plantio, contendo os macronutrientes nitrogênio $(\mathrm{N})$, fósforo $(\mathrm{P})$ e potássio $(\mathrm{K})$ na proporção de $3 \mathrm{~g}$ de cada elemento por planta.

\subsection{Análises dos dados}

Os dados obtidos foram submetidos à análise de regressão ao nível de $5 \%$ e $1 \%$ de probabilidade, por meio da utilização do software de análise estatística SISVAR, versão 5.3 beta [14]. Os pressupostos de normalidade e homogeneidade dos dados foram avaliados pelos testes Kolmogorov-Smirnov e Bartlett, respectivamente, e possíveis modelos de regressão, gerados no Microsoft Office Excel.

\section{Resultados e discussões}

\subsection{Resultados dos testes de normalidade e homogeneidade}

Somente os dados referentes à altura das mudas e ao comprimento das primeiras brotações emitidas não atenderam os pressupostos de normalidade e homogeneidade. Nesse caso, foram submetidos à transformação Raiz Quadrada $=Y_{i j}=\sqrt{ } Y_{i j}, e$ novamente testados, desta vez, atendendo ambos.

\subsection{Resultados da análise de regressão}

Conforme os resultados da análise de regressão apresentados na Tabela 1, os parâmetros analisados na parte aérea das mudas, a altura $(\mathrm{P} \leq 0,01)$, o comprimento do primeiro broto $(\operatorname{Pr} \leq 0,01)$, o diâmetro do primeiro broto $(\mathrm{P} \leq 0,05)$ e $\mathrm{O}$ comprimento do segundo broto $(\mathrm{P} \leq 0,01)$, responderam linearmente aos comprimentos das estacas utilizadas para a formação das mudas sob ambiente protegido. Porém, em relação ao número de brotações emitidas, houve uma reação diferente, sendo o modelo matemático que melhor explica esse comportamento o polinomial de $2^{\circ}$ grau $(\mathrm{P} \leq 0,01)$.

A relação linear positiva entre os parâmetros biométricos analisados em que o incremento foi avaliado por medições, no caso, da altura (Figura 1), do comprimento do primeiro broto (Figura 3), do diâmetro do primeiro broto (Figura 4), e do comprimento do segundo broto (Figura 5), em função do comprimento da estaca pode ser explicada pelo fato de mudas formadas a partir de estacas de maiores comprimentos possuírem maiores reservas e disponibilidade de nutrientes armazenados [15], principalmente carboidratos, o que contribui diretamente para a formação e o crescimento de brotos e raízes [16]. 


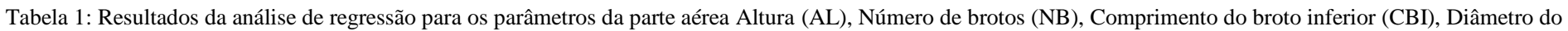
broto inferior (DBI) e Comprimento do segundo broto (CSB).

\begin{tabular}{|c|c|c|c|c|c|c|c|c|c|c|c|}
\hline \multirow{2}{*}{$\mathrm{CV}$} & \multirow{2}{*}{ GL } & \multicolumn{2}{|c|}{$\mathrm{AL}(\mathbf{c m})$} & \multicolumn{2}{|c|}{ NB } & \multicolumn{2}{|c|}{ CPB (cm) } & \multicolumn{2}{|c|}{ DPB (cm) } & \multicolumn{2}{|c|}{$\mathrm{CSB}(\mathbf{c m})$} \\
\hline & & $\mathrm{Fc}$ & $\mathrm{P}>\mathrm{F}$ & $\mathrm{Fc}$ & $\mathrm{P}>\mathrm{F}$ & $\mathrm{Fc}$ & $\mathrm{P}>\mathrm{F}$ & $\mathrm{Fc}$ & $\mathrm{P}>\mathrm{F}$ & $\mathrm{Fc}$ & $\mathrm{P}>\mathrm{F}$ \\
\hline Linear & 1 & $53,963^{* *}$ & $0,000^{* *}$ & $4,049^{*}$ & $0,048^{*}$ & $7,072^{* *}$ & $0,010^{* * *}$ & $5,785^{*}$ & $0,019^{*}$ & $8,237^{* *}$ & $0,005^{* *}$ \\
\hline Polinomial2 & 1 & $0,148^{\mathrm{ns}}$ & $0,702^{\mathrm{ns}}$ & $8,034^{* *}$ & $0,006^{* *}$ & $1,858^{\mathrm{ns}}$ & $0,177^{\mathrm{ns}}$ & $1,362^{\mathrm{ns}}$ & $0,247^{\mathrm{ns}}$ & $0,449^{\mathrm{ns}}$ & $0,505^{\mathrm{ns}}$ \\
\hline Desvio & 2 & $2,478^{\mathrm{ns}}$ & $0,091^{\mathrm{ns}}$ & $1,075^{\mathrm{ns}}$ & $0,346^{\mathrm{ns}}$ & $2,165^{\mathrm{ns}}$ & $0,122^{\mathrm{ns}}$ & $0,575^{\mathrm{ns}}$ & $0,565^{\text {ns }}$ & $2,301^{\mathrm{ns}}$ & $0,107^{\mathrm{ns}}$ \\
\hline Erro & 75 & & & & & & & & & & \\
\hline
\end{tabular}

${ }^{* *}$ Significativo a $1 \%$ de probabilidade de erro $(\operatorname{Pr} \leq 0,01)$

Significaivo a 5\% de probabilidade de erro $(\operatorname{Pr} \leq 0,05)$

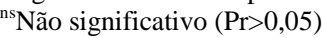

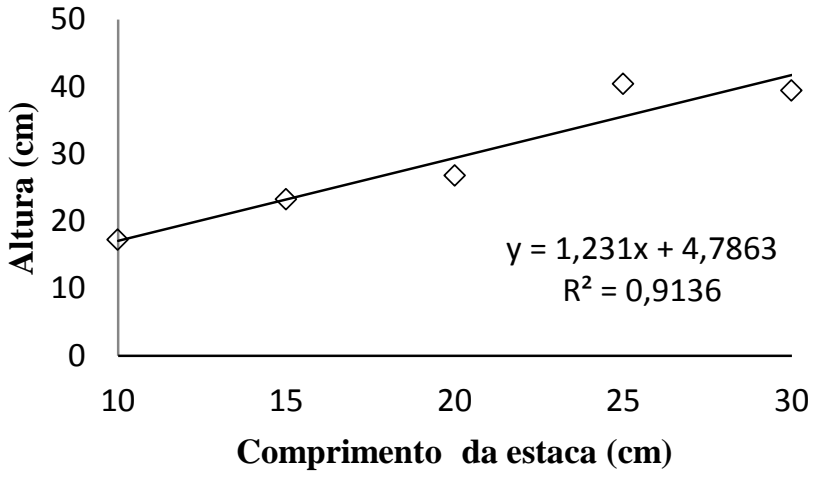

Figura 1: Modelo linear para a Altura (AL) em função do comprimento das estacas utilizadas para a formação das mudas sob ambiente protegido.

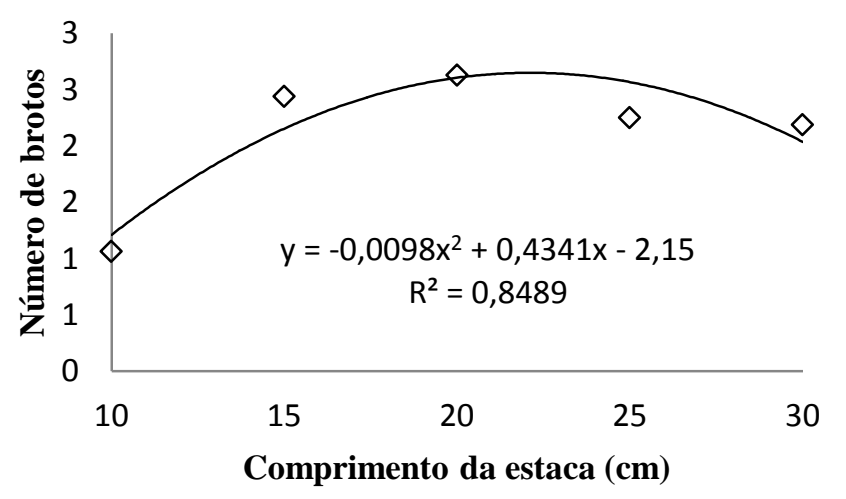

Figura 2: Modelo polinomial de segundo grau para o número de brotos emitidos (NB) em função do comprimento das estacas utilizadas para a formação das mudas sob ambiente protegido.

O número de brotações emitidas em relação ao comprimento da estaca utilizada para a formação das mudas sob ambiente protegido (Figura 2) teve o ponto de máxima emissão em $24,11 \mathrm{~cm}$, conforme derivada primeira da equação em questão, na qualas mudas poderiam emitir, aproximadamente 3,08 brotos. Esse ponto ficou próximo ao comprimento das estacas utilizadas no tratamento quatro, que tinham $25 \mathrm{~cm}$, menores que as do tratamento cinco, que possuíam $30 \mathrm{~cm}$. Tais resultados contrastam com os obtidos por [17], que analisaramo número de brotações emitidas por estacas de pitaia, com tamanhos de $50 \mathrm{~cm}$ e $100 \mathrm{~cm}$. Na ocasião, os pesquisadores não identificaram diferença significativa entre o número de brotações emitidas, ou seja, mesmo as estacas maiores possuindo o dobro do tamanho das menores, não emitiram mais brotações do que as primeiras. Esse resultado comprova a eficácia da utilização de comprimentos menores de estaca para a formação de pomares de pitaia sem comprometimento do número de emissões de brotações [17].

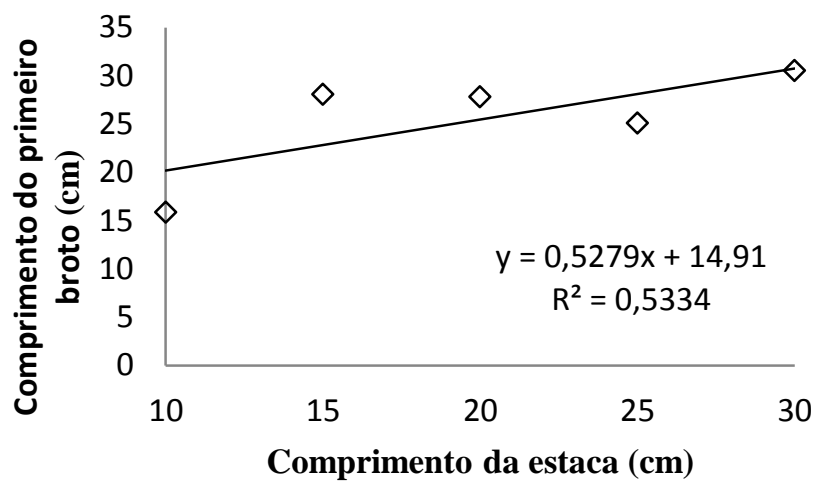

Figura 3: Modelo linear para o comprimento do primeiro broto (CPB) em função do comprimento das estacas utilizadas para a formação das mudas sob ambiente protegido.

De acordo com os resultados, asmudas formadas a partir de estacas de maior comprimento tiveram maior incremento em altura, comprimento e diâmetro das brotações emitidas. Em relação ao número de brotações, os resultados corroboram com [18], que, analisando o efeito do comprimento das estacas utilizadas para a produção de mudas de pitaia sob telado e doses 
de ácido indolbutírico (AIB), recomendam a utilização de estacas de $25 \mathrm{~cm}$.

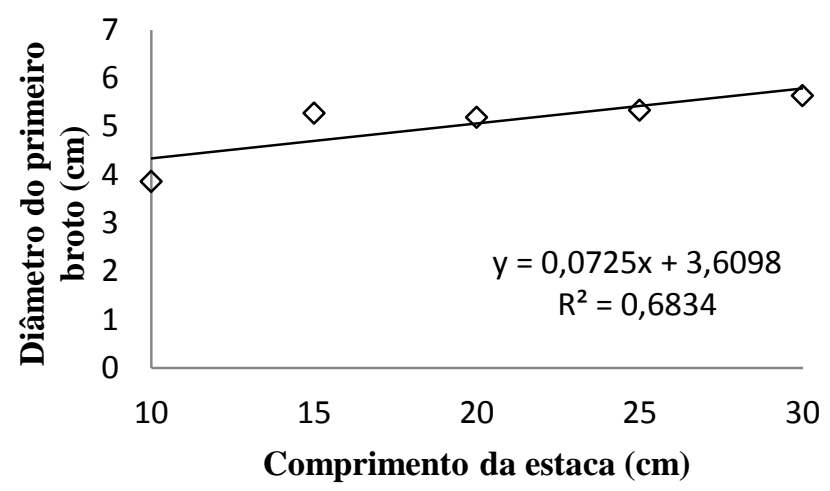

Figura 4: Modelo linear para o diâmetro do primeiro broto (DPB) em função do comprimento das estacas utilizadas para a formação das mudas sob ambiente protegido.

De maneira geral, os resultados obtidos, abrem a possibilidade da utilização de menores comprimentos de estacas, principalmente, na ocasião onde há escassez de material propagativo, já que o ponto de máxima emissão de brotações se deu próximo às estacas de $25 \mathrm{~cm}$. No entanto, se a ocasião for de abundância, estacas maiores podem proporcionar incrementos superiores em altura, diâmetro e comprimento das brotações emitidas.

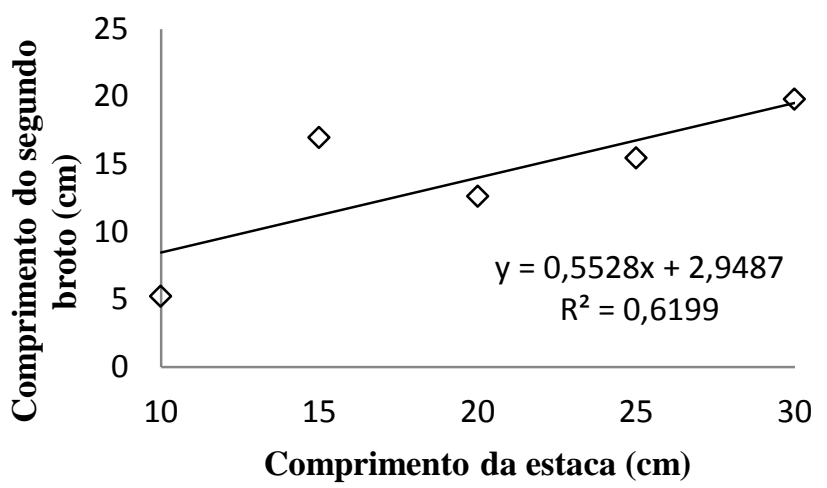

Figura 5: Modelo linear para o comprimento do segundo broto (CSB) em função do comprimento das estacas utilizadas para a formação das mudas sob ambiente protegido.

\section{Conclusões}

O comprimento das estacas influenciou o ganho em altura, comprimento e diâmetro das brotações emitidas, apresentando uma relação positiva linear, ou seja, quanto maior a estaca, maiores os incrementos.

Estacas de $25 \mathrm{~cm}$, podem ser utilizadas para a produção de mudas sob ambiente protegido sem o comprometimento do número de emissões de brotações.
O ponto de máxima emissão de brotações se deu em $24,11 \mathrm{~cm}$, quando as mudas poderiam emitir, aproximadamente, três brotações.

\author{
Agradecimentos \\ À Universidade Federal Rural da Amazônia.
}

\section{DETERMINATION OF CUTTINGSLENGTH TO PRODUCE PITAYA SEEDLINGS (Hylocereus costaricensis) IN A PROTECTED} ENVIRONMENT

ABSTRACT: The paper aimed to find the best length of the cuttings for the production of pitaya (Hylocereus costaricensis) seedlings in a protected environment. The experiment was carried out at the Federal Rural University of Amazônia, Campus Capitão Poço - Pará, in a completely randomized design with five treatments and four replicates, each treatment consisting of a length of cuttings: $10 \mathrm{~cm}, 15 \mathrm{~cm}, 20 \mathrm{~cm}, 25 \mathrm{~cm}$ and $30 \mathrm{~cm}$, forming 20 experimental plots. Each of these plots was composed of four cuttings, totaling 80 seedlings. With the appearance of the first shoots, the following parameters were evaluated: seedling height in $\mathrm{cm}(L A)$, number of shoots $(N B)$, length and diameter of the first shoot $(C P B$ and $D B I)$ and length of the second shoot $(C S B)$. The results obtained prove the effectiveness of the use of shorter lengths of stakes, especially when there is a lack of propagation material, since the point of maximum emission of shoots was close to $25 \mathrm{~cm}$ cuttings. However, if there is abundance, larger cuttings may provide higher increments in height, diameter and length of shoots emerged. The length of the cuttings has influenced the gain in height, length and diameter of the shoots emerged, presenting a linear positive relation, that is, the bigger the cutting, the greater the increments. $25 \mathrm{~cm}$ cuttings can be used to produce seedlings without compromising the number of sprouts emerged. The point of maximum emergence of shoots occurred in $24.11 \mathrm{~cm}$, when the seedlings could develop approximately three shoots.

Keywords: Vegetative propagation, Cladodium, Cactaceae, CAM metabolism.

\section{Referências}

[1] JUNQUEIRA, K. P.; JUNQUEIRA, N. T. V.; RAMOS, J. D.; PEREIRA, A V. Informações preliminares sobre uma espécie de Pitaya do Cerrado. Documentos/ EMBRAPA Cerrados, Ed. 1. Planaltina, DF, 18 p. 2002.

[2] MIZRAHI, Y.; NERD, A.; NOBEL, P. S. Cacti as crops. Horticultural Review, New York, v. 18, p. 291-320, 1997.

[3] ORTIZ, H. Y. D.; LIVERA, M. M. La pitahaya (Hylocereus spp): Recurso genético de América. PIMIENTA B. et al. (ed.s). Memorias del $6^{\circ}$ Congreso Nacional y $4^{\circ}$ Internacional sobre El conocimiento y aprovechamientodelnopal. Guadalajara. México. p. 191-194, 1995. 


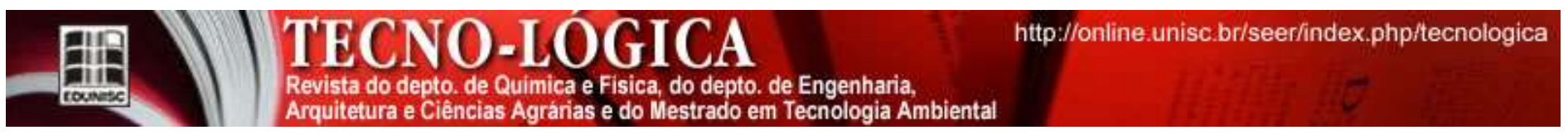

[4] DONADIO, L. C. Pitaya. Revista Brasileira de Fruticultura, Jaboticabal, v. 31, n. 3, p. 637-929, 2009.

[5] SILVA, A. C. C.; MARTINS, A. B. G.; CAVALlARI, L. L. Qualidade de frutos de pitaya em função da época de polinização, da fonte de pólen e da coloração da cobertura. Revista Brasileira de Fruticultura, v. 33, n. 4, p. 11621168,2011

[6] ZEE, F.; YEN, CHUNG-RUEN; NISHINA, M. Pitaya (Dragon fruit, Straw berry pearl). Fruits e Nuts, Hawai, n. 9, pag. 1-3, 2004.

[7] LE BELLEC, F.; VILLANT, F.; IMBERT, E. Pitahaya (Hylocereus spp.): a new crop, a market with a future. Fruits, France, v. 61, n. 4, p. 237 -250. 2006.

[8] NERD, A.; TEL-ZUR, N.; MIZRAHI, Y. Fruit of vine and columnar cacti. In: NOBEL, P. S. (Ed.). Cacti: biology and uses. Los Angeles: UCLA, p. 254$262,2002$.

[9] JUNQUEIRA, K. P.; FALEIRO, F. G.; BELLON, G.; JUNQUEIRA, N. T. V.; LIMA, C. A.; FONSECA, K. G.; SANTOS E. C. Variabilidade genética de acessos de pitaya com diferentes níveis de produção por meio de marcadores RAPD. IX Simpósio Nacional do Cerrado. II Encontro Internacional Savanas Tropicais. 2008

[10] MARQUES, V. B. et al. Fenologia reprodutiva de pitaia vermelha no município de Lavras, MG. Ciência Rural, Santa Maria, v.41, n.6, p.984-987, 2011.

[11] BRAGA, M. F. et al. Enraizamento de estacas de três espécies silvestres de Passiflora. Revista Brasileira de Fruticultura, v. 28, n. 2, p. 284-288, 2006.

[12] COSTA, L. C. do B.; PINTO, J. E. B. P.; BERTOLUCCI, S. K. V. Comprimento da estaca e tipo de substrato na propagação vegetativa de atroveran . Ciência Rural, v. 37, n. 4, p. 1157- 1160, 2007.

[13] LIMA, C. A. de. Caracterização, propagação e melhoramento genético de pitaya comercial e nativa do cerrado. 2013. 124 f. Tese (Doutorado em Agronomia) - Universidade de Brasília, Brasília, 2013.

[14] FERREIRA, D. F. SISVAR - Sistema de análise de variância. Versão 5.3. Lavras-MG: UFLA, 2010.

[15] DELGADO, J. P. M.; YUYAMA, K. Comprimento de estaca de camu-camu com ácido indolbutírico para a formação de mudas. Revista Brasileira de Fruticultura, Jaboticabal, v. 32, n. 2, p. 522-526, 2010.

[16] NICOLOSO, F. T.; CASSOL, L. F.; FORTUNATO, R. P. Comprimento da estaca de ramo no enraizamento de ginseng brasileiro (Pfaffia glomerata). Ciência Rural, Santa Maria, v.31, n.1, p.57-60, 2001.

[17] LÓPEZ, R. G; DÍAZ, J. P.; FLORES, G. M. Vegetative propagation of thre especies of cacti: pitaya, Tunillo and jiotilla. Agrociência, vol 34, n. 3, p. 363$367,2000$.

[18] BASTOS, D. C. et al. Propagação da pitaya 'vermelha' por estaquia. Ciência e Agrotecnologia, Lavras, v. 30, n. 6, p. 1106-1109, 2000. 